\title{
Synthesis of $\alpha$-Tropolones through Autoxidation of Dioxole-Fused Cycloheptatrienes
}

Alex J. Berkowitz ${ }^{1,2}$ and Ryan P. Murelli ${ }^{1,2,3^{*}}$

${ }^{I}$ Department of Chemistry, Brooklyn College, The City University of New York, Brooklyn College, Brooklyn, NY ${ }^{2}$ Ph.D. Program in Chemistry, The Graduate Center of The City University of New York, New York, NY

${ }^{3}$ Ph.D. Program in Biochemistry, The Graduate Center of The City University of New York, New York, NY

\section{Supporting Information}

\section{Table of Contents}

\section{I. ${ }^{1} \mathrm{H}$ and ${ }^{13} \mathrm{C}$ NMR Spectra}

Ethyl 6H-cyclohepta[d][1,3]dioxole-6-carboxylate (2).................................................................................

Ethyl 5H-cyclohepta[d][1,3]dioxole-5-carboxylate (3)...............................................................................

Ethyl 3,4-dimethoxybicyclo[4.1.0]hepta-2,4-diene-7-carboxylate (8)....................................................................5-5

Ethyl 2,3-dihydro-7H-cyclohepta[b][1,4]dioxine-7-carboxylate (11) ................................................................ 6

Ethyl 2,2-dimethyl-6H-cyclohepta[d][1,3]dioxole-6-carboxylate (13)...............................................................s-8

Ethyl 5-methoxy-6H-cyclohepta[d][1,3]dioxole-6-carboxylate (24)..................................................................s-10

Ethyl 4-hydroxy-5-oxocyclohepta-1,3,6-triene-1-carboxylate (5)................................................................... 12

Ethyl 4-hydroxy-2-methoxy-5-oxocyclohepta-1,3,6-triene-1-carboxylate (25).......................................................

4-hydroxy-5-oxocyclohepta-1,3,6-triene-1-carboxylic acid (20).......................................................................

N-butyl-4-hydroxy-5-oxocyclohepta-1,3,6-triene-1-carboxamide (21a).......................................................... 20

N-(2-(3-(but-3-yn-1-yl)-3H-diazirin-3-yl)ethyl)-4-hydroxy-5-oxocyclohepta-1,3,6-triene-1-carboxamide (21b)...s-22

N-benzyl-4-hydroxy-5-oxocyclohepta-1,3,6-triene-1-carboxamide (21c)…....................................................... 24

2,4-dihydroxy-5-oxocyclohepta-1,3,6-triene-1-carboxylic acid (26).................................................................. 26

Ethyl 4-hydroxy-3-oxocyclohepta-1,4,6-triene-1-carboxylate (6)........................................................................2-28 
Ethyl $6 H$-cyclohepta $[d][1,3]$ dioxole-6-carboxylate (2)

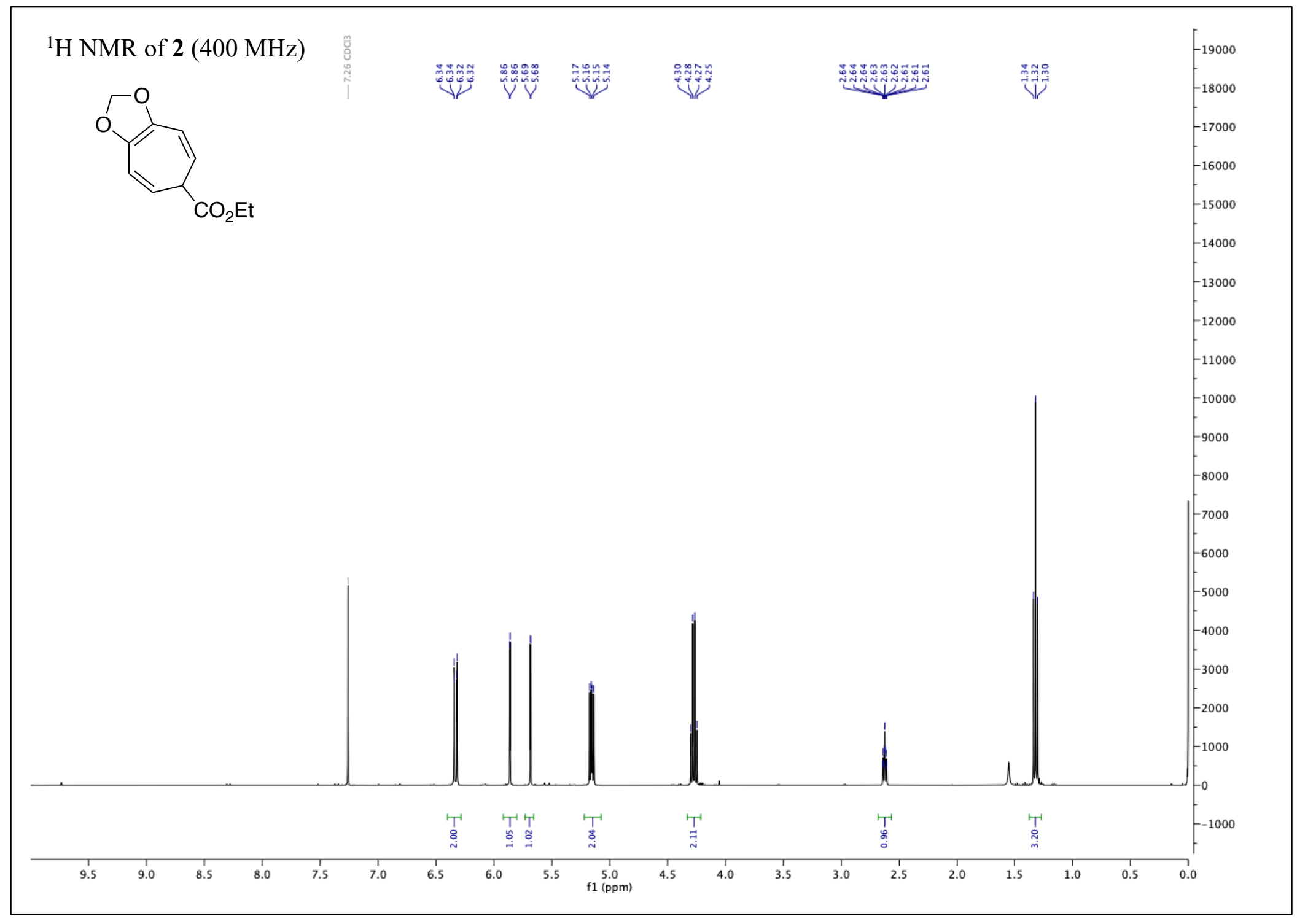


Ethyl $5 H$-cyclohepta $[d][1,3]$ dioxole-5-carboxylate (3)

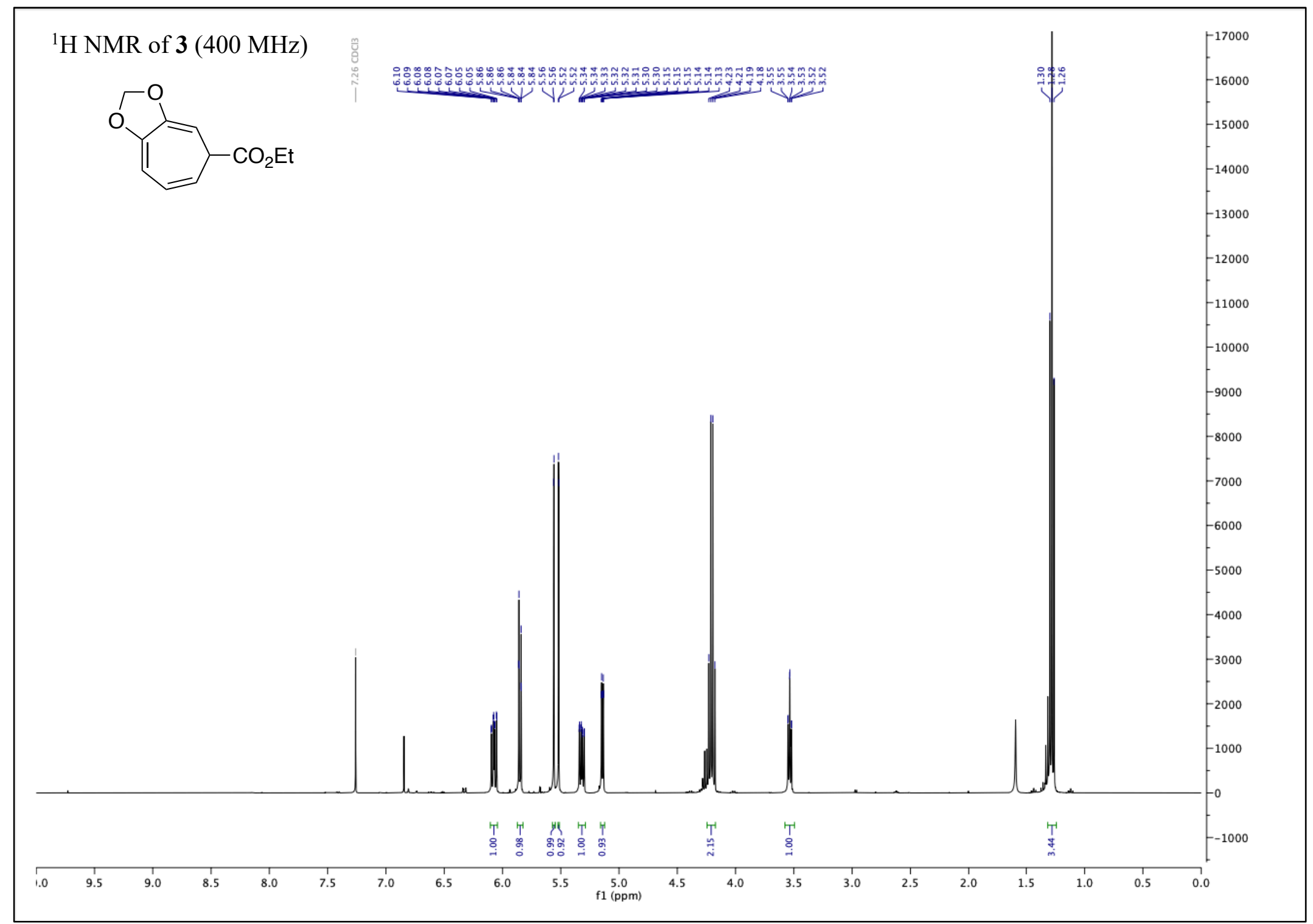




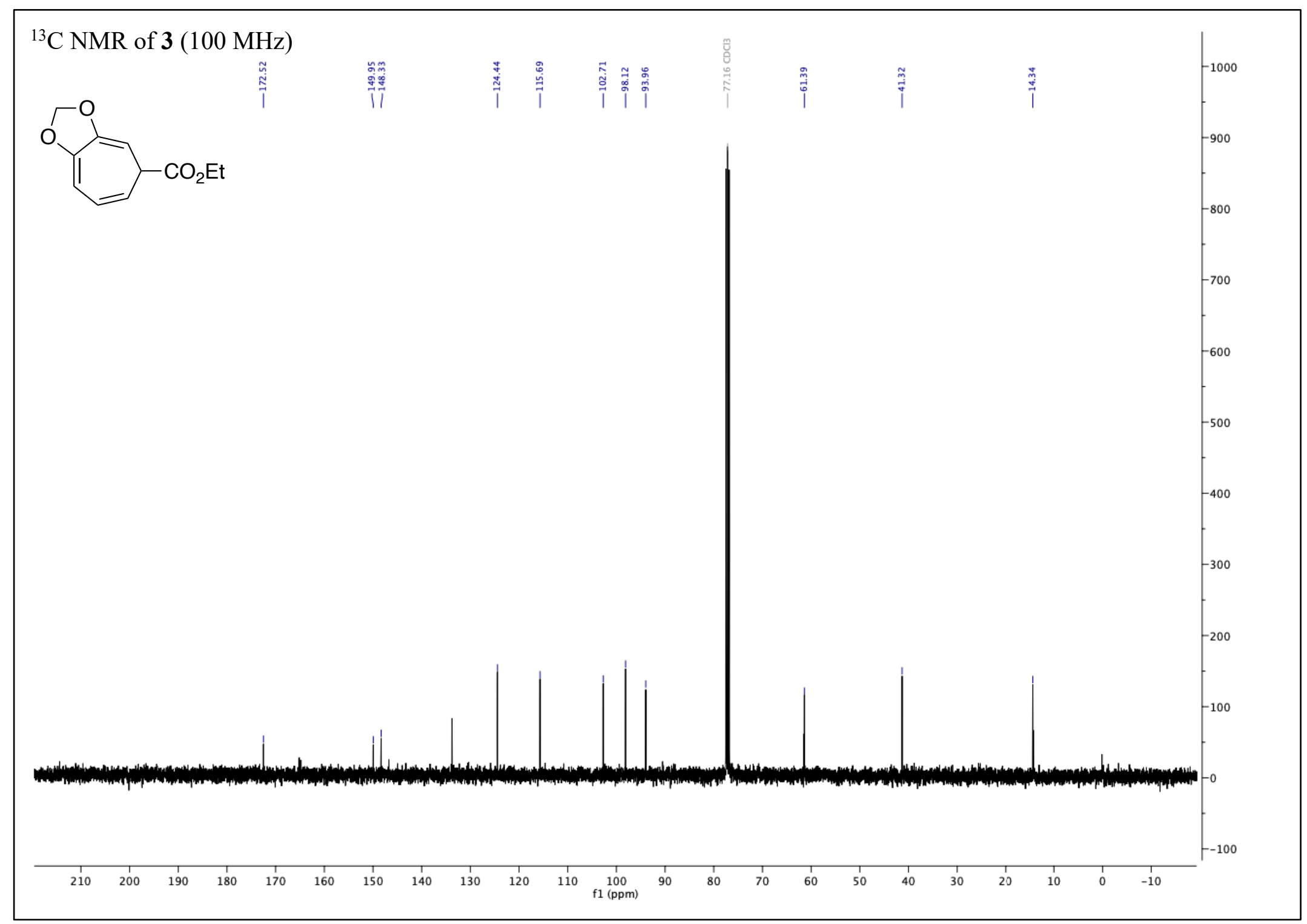


Ethyl 3,4-dimethoxybicyclo[4.1.0]hepta-2,4-diene-7-carboxylate (8)

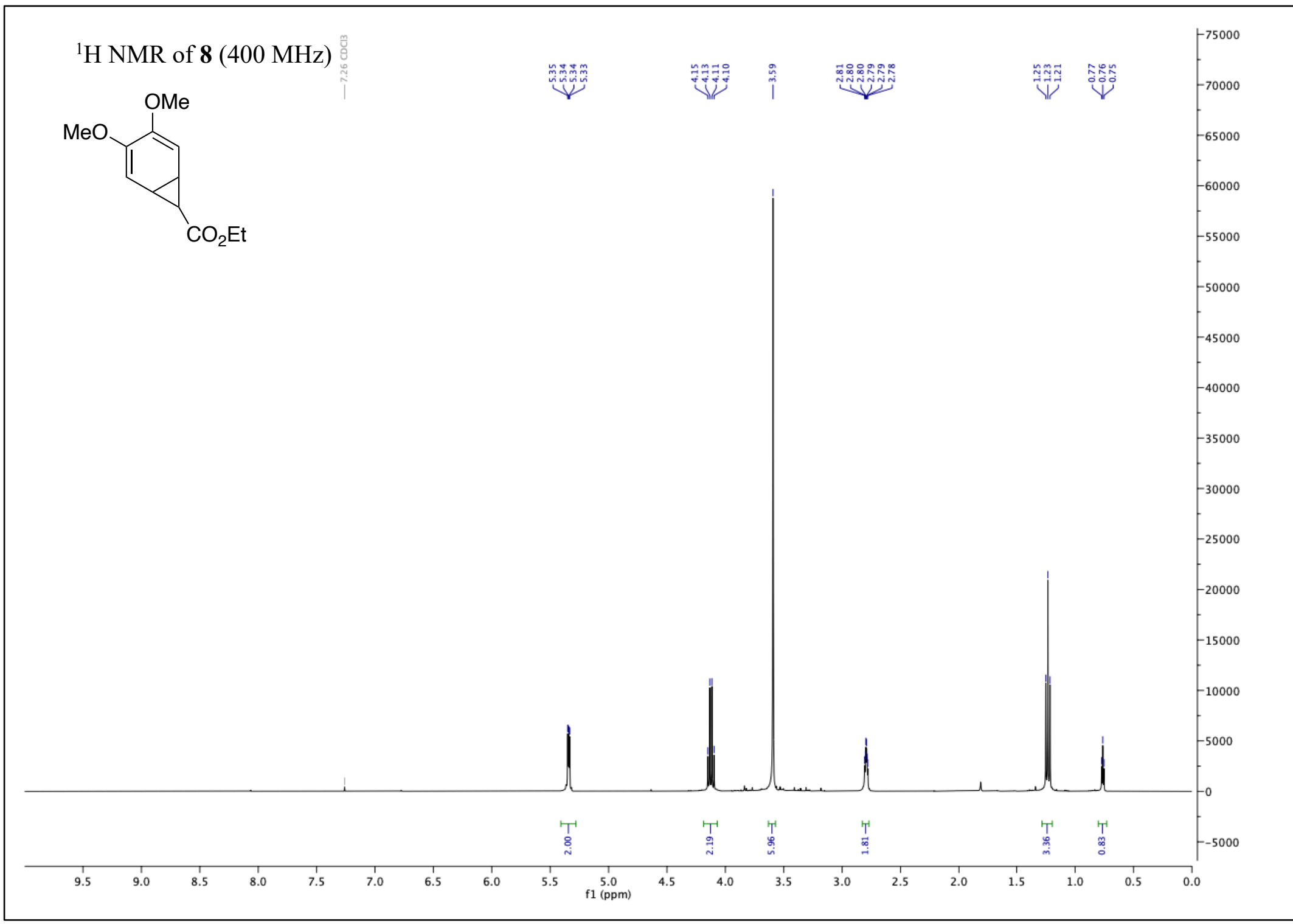


Ethyl 2,3-dihydro-7H-cyclohepta[b][1,4]dioxine-7-carboxylate (11)

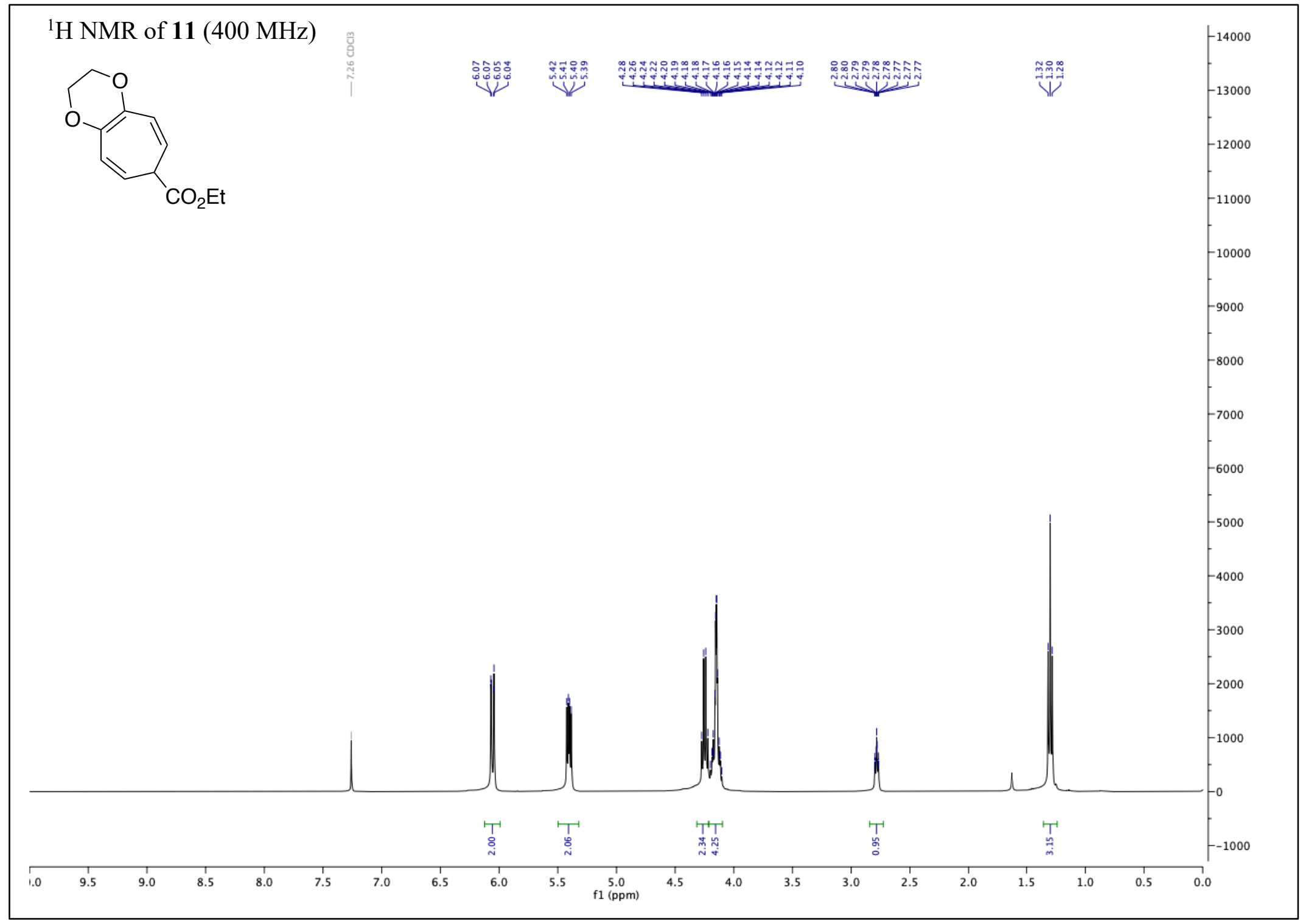




$$
\text { S }
$$


Ethyl 2,2,-dimethyl-6H-cyclohepta[ $d][1,3]$ dioxole-6-carboxylate (13)

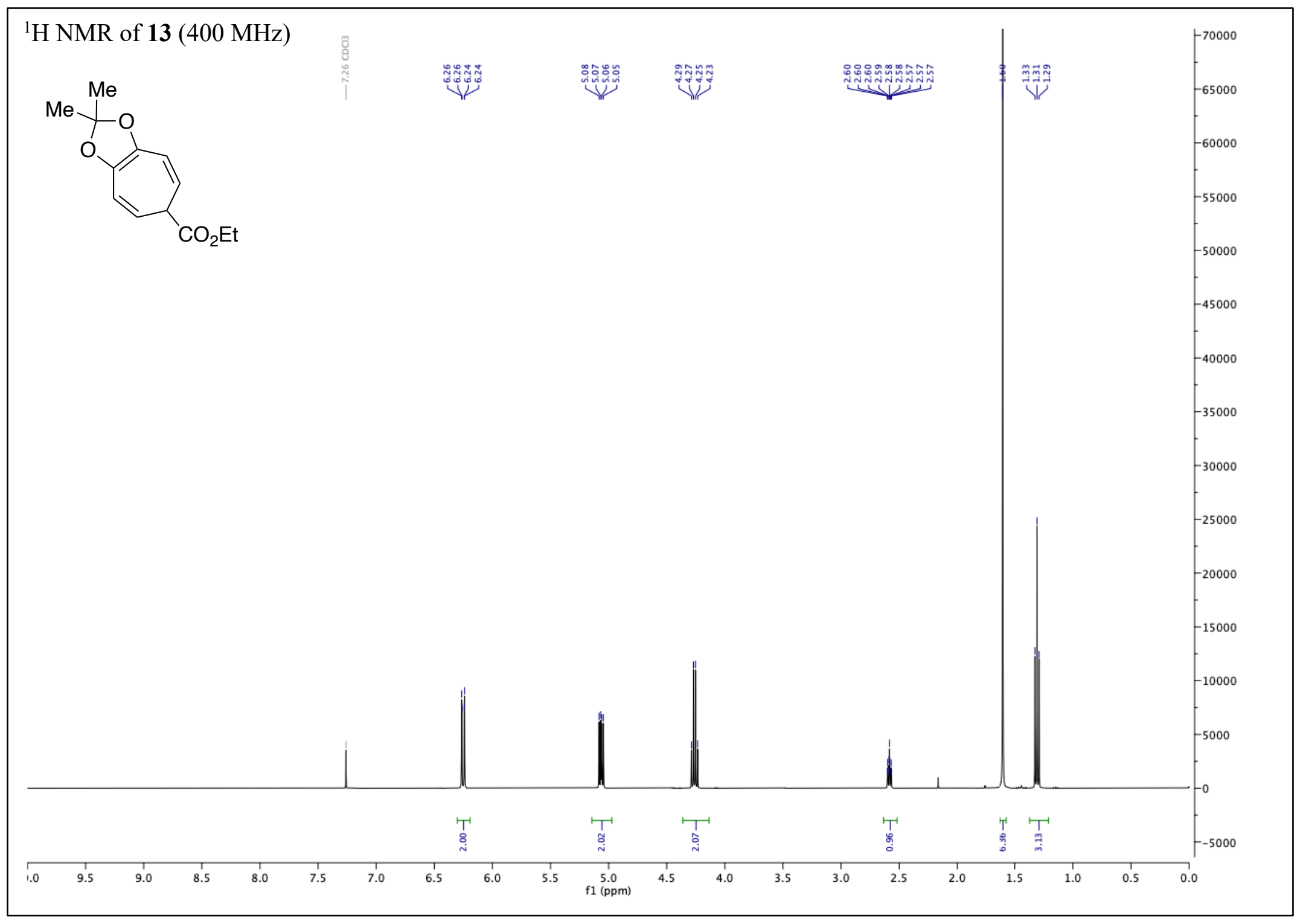




$$
+
$$


Ethyl 5-methoxy-6H-cyclohepta $[d][1,3]$ dioxole-6-carboxylate (24)

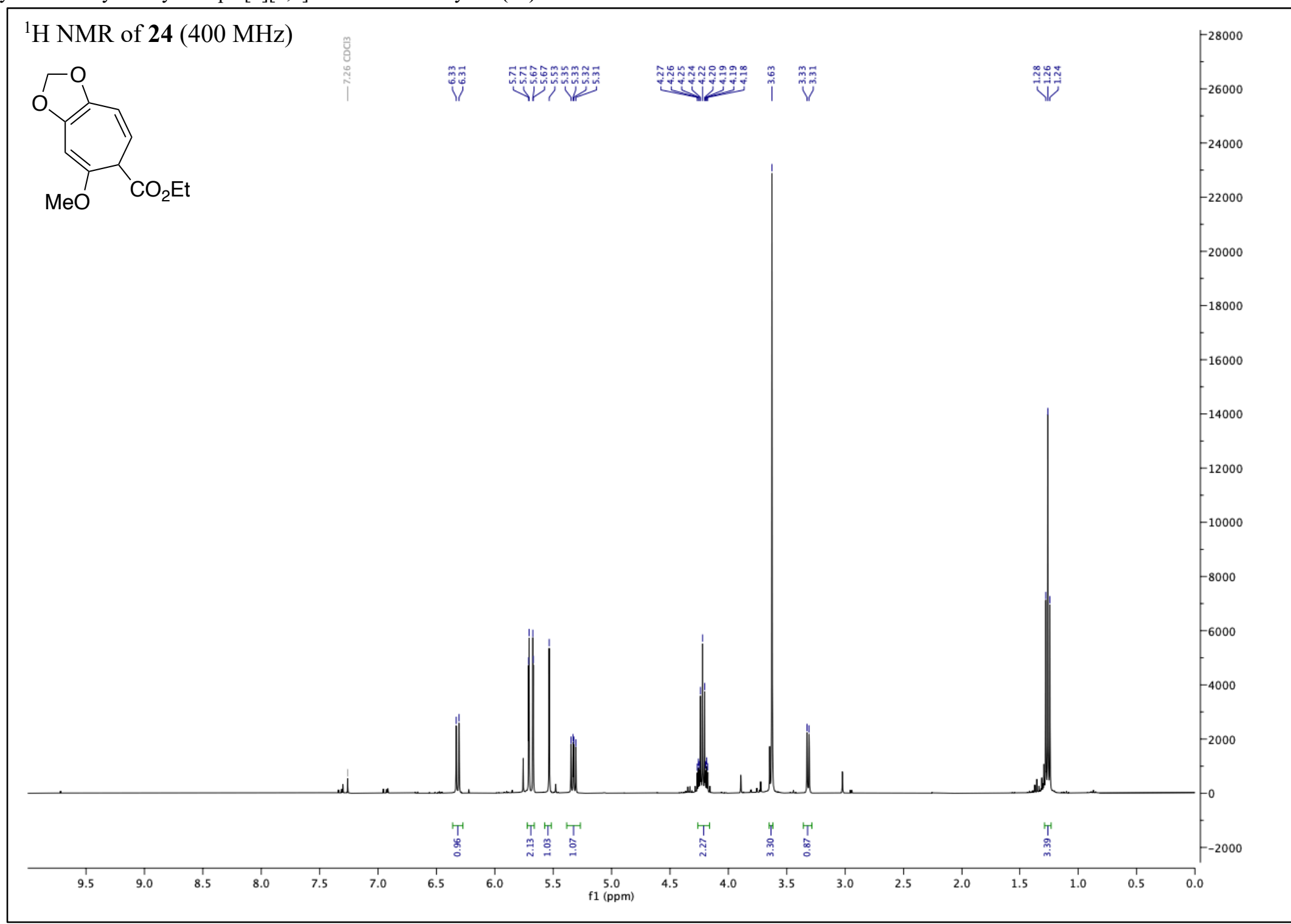

$s-10$ 

Ethyl 4-hydroxy-5-oxocyclohepta-1,3,6-triene-1-carboxylate (5) (Via CHT 3)

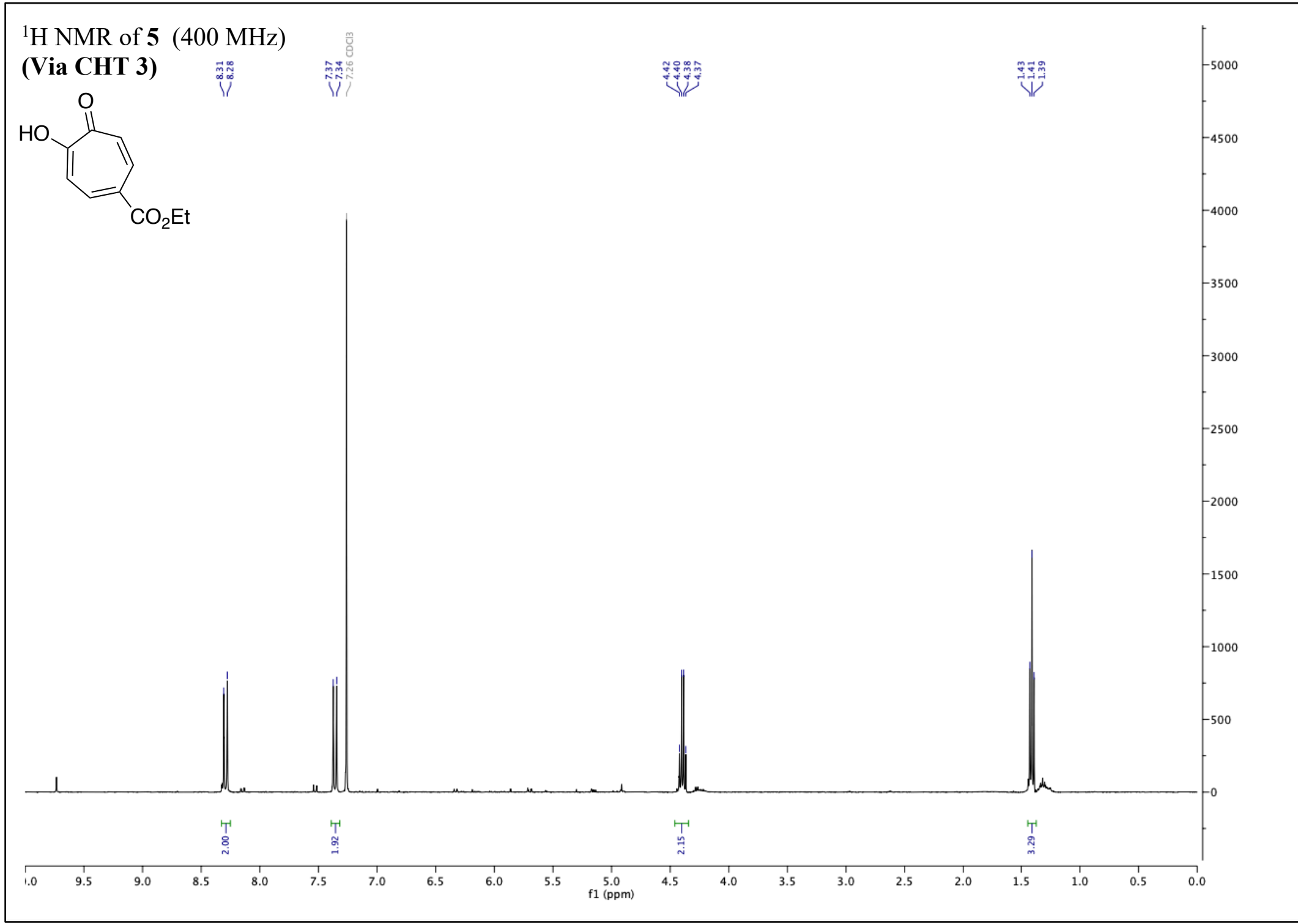




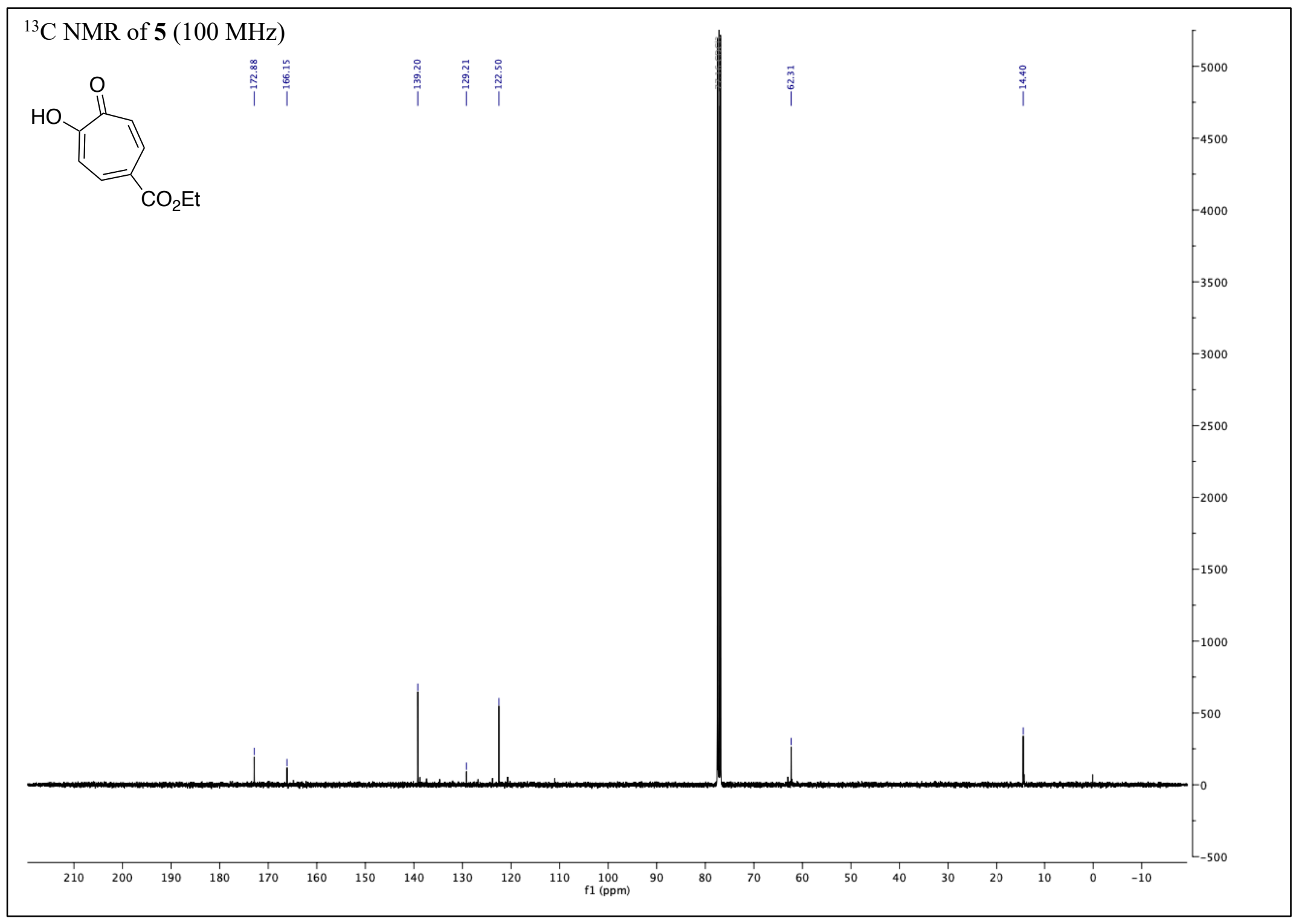


Ethyl 4-hydroxy-5-oxocyclohepta-1,3,6-triene-1-carboxylate (5) (Large scale reaction)

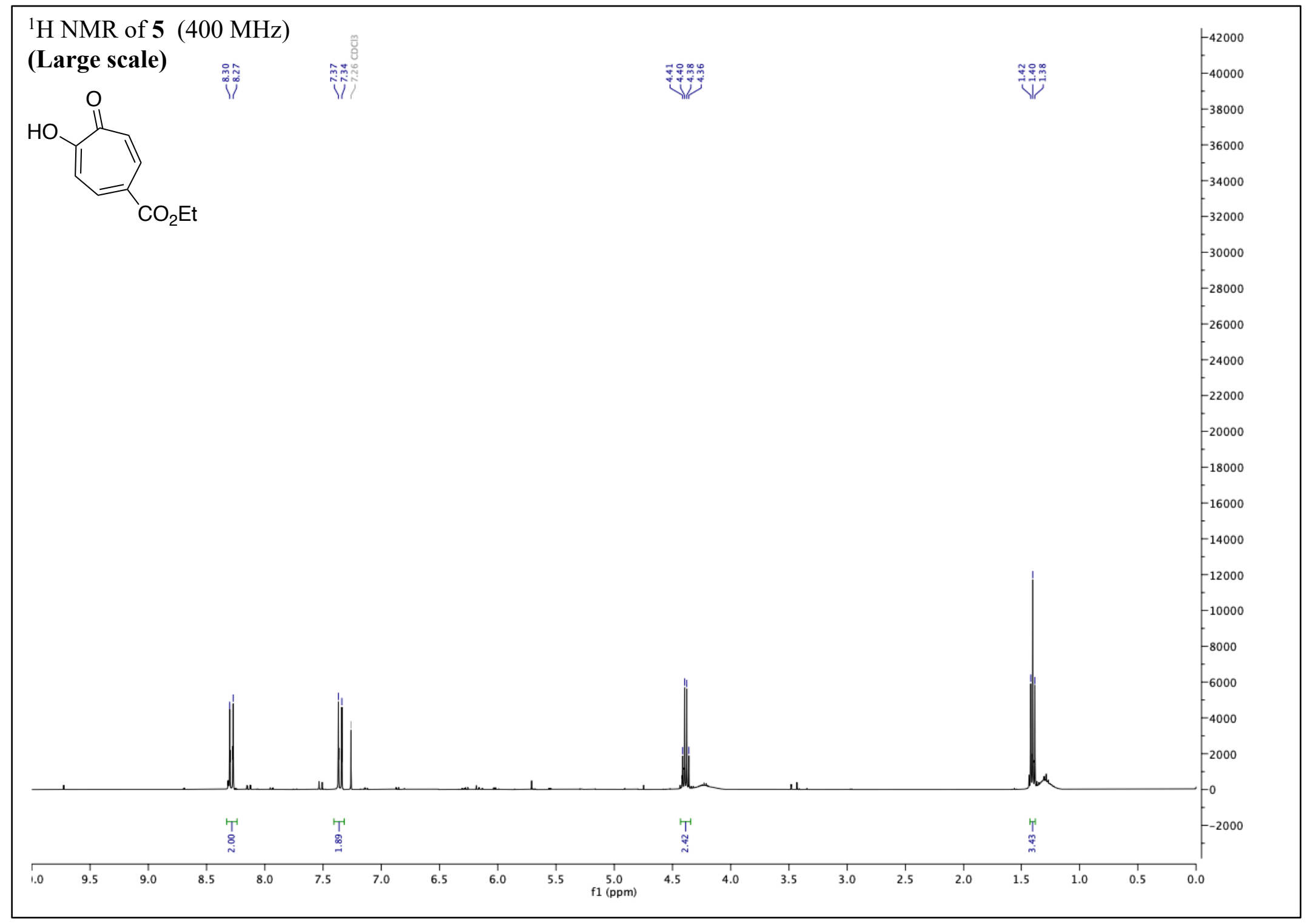


Ethyl 4-hydroxy-5-oxocyclohepta-1,3,6-triene-1-carboxylate (5) (Via acetonide CHT 13)

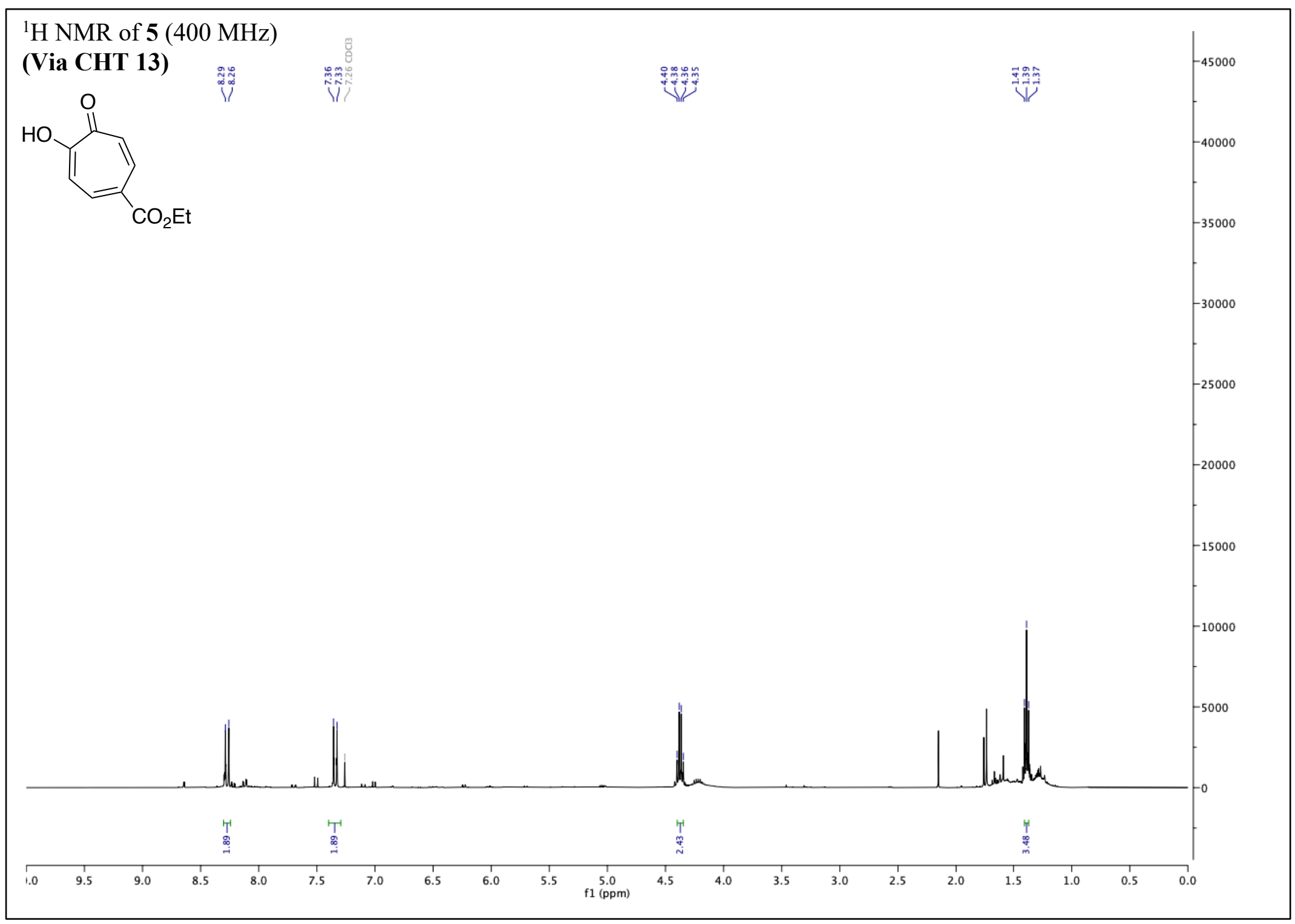


Ethyl 4-hydroxy-2-methoxy-5-oxocyclohepta-1,3,6-triene-1-carboxylate (25)

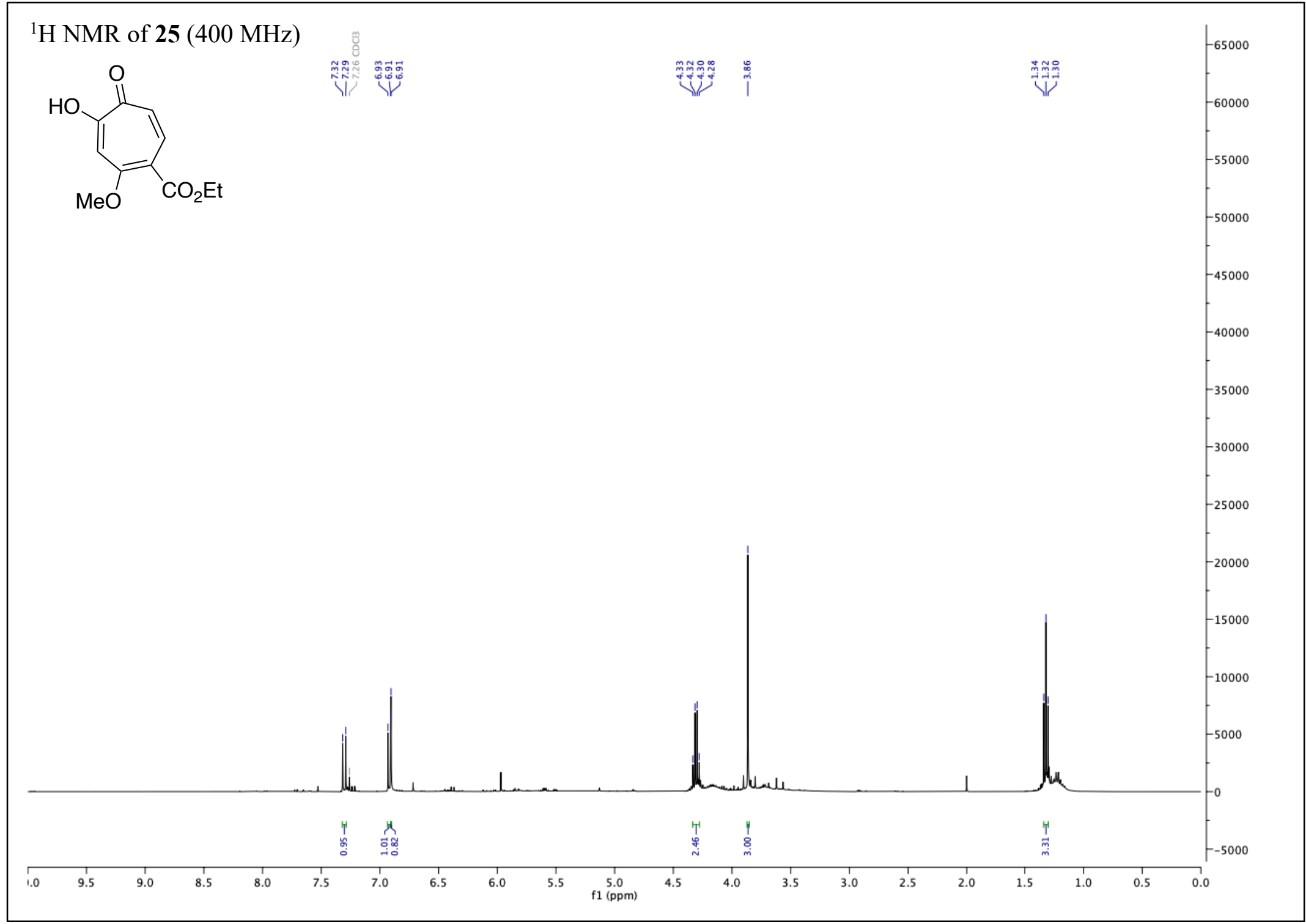

$s-16$ 


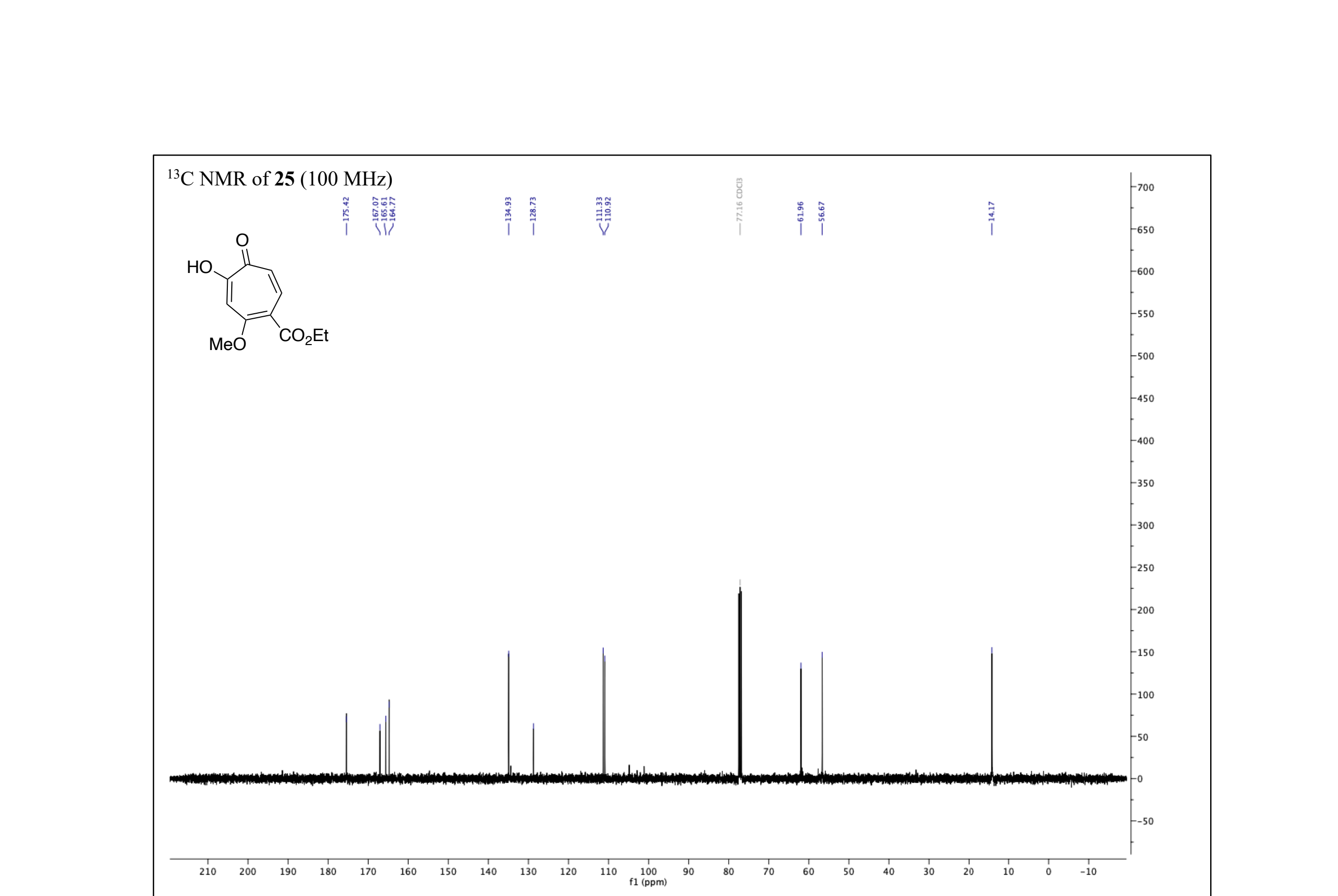


4-hydroxy-5-oxocyclohepta-1,3,6-triene-1-carboxylic acid (20)

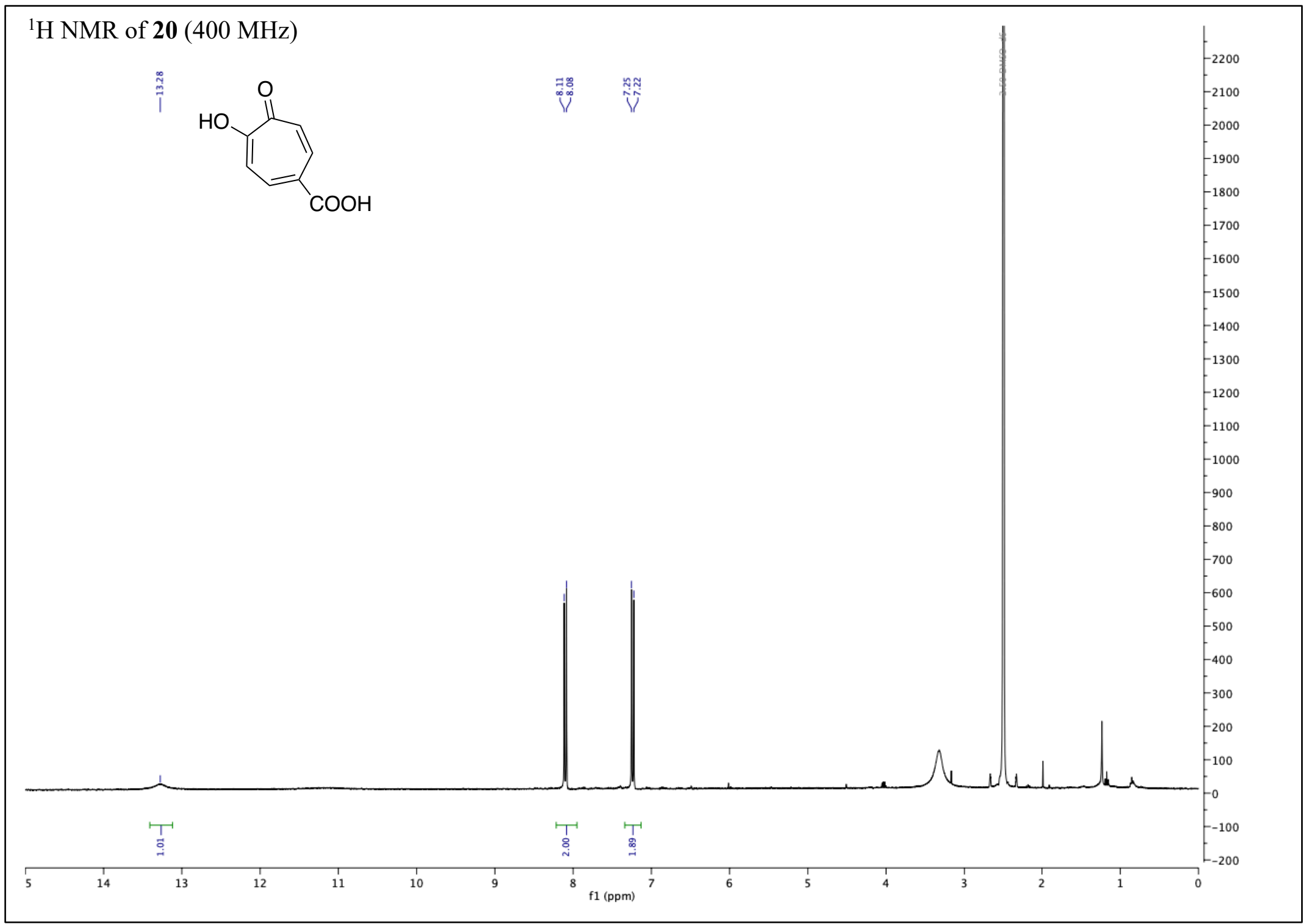




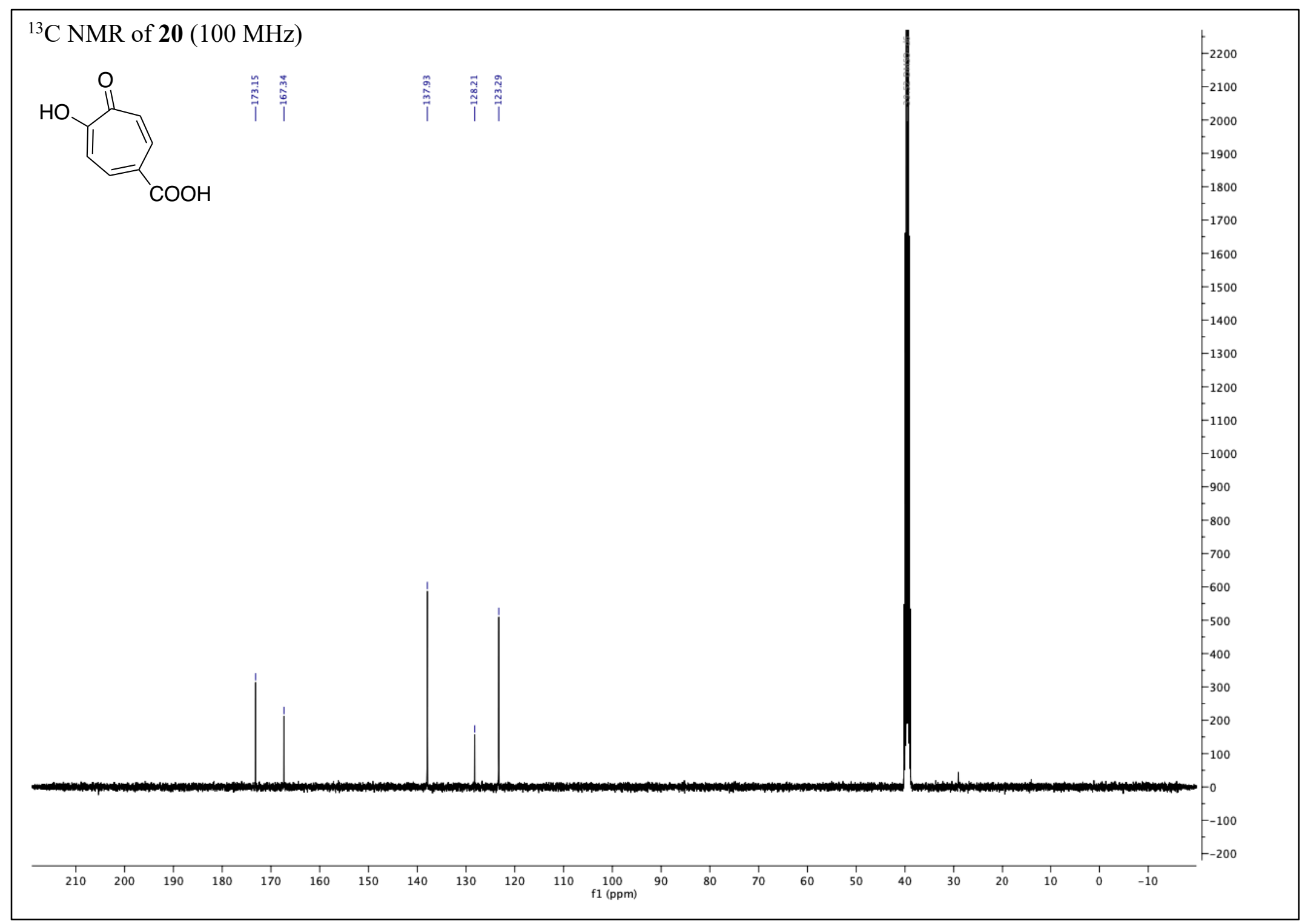


$N$-butyl-4-hydroxy-5-oxocyclohepta-1,3,6-triene-1-carboxamide (21a)

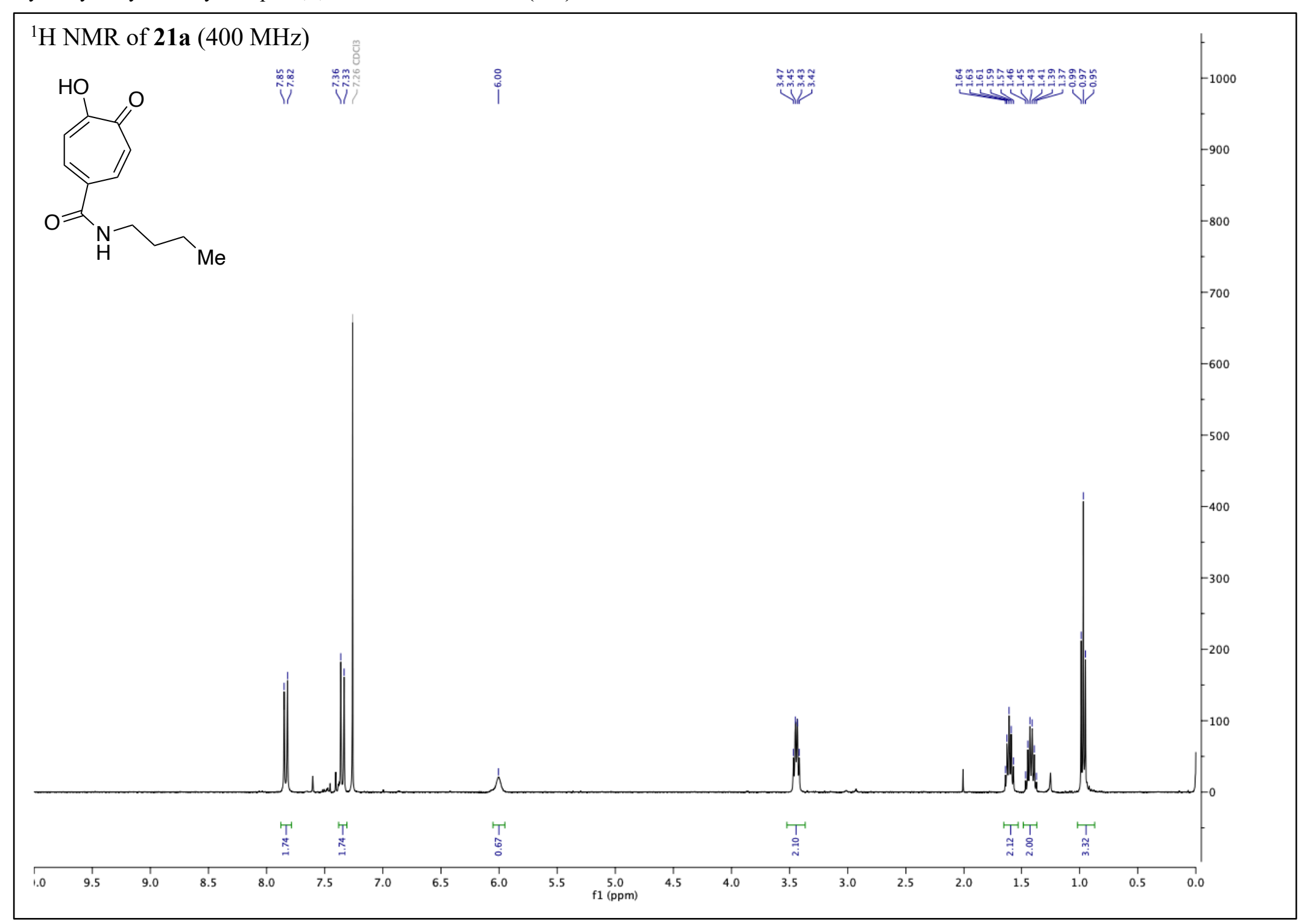




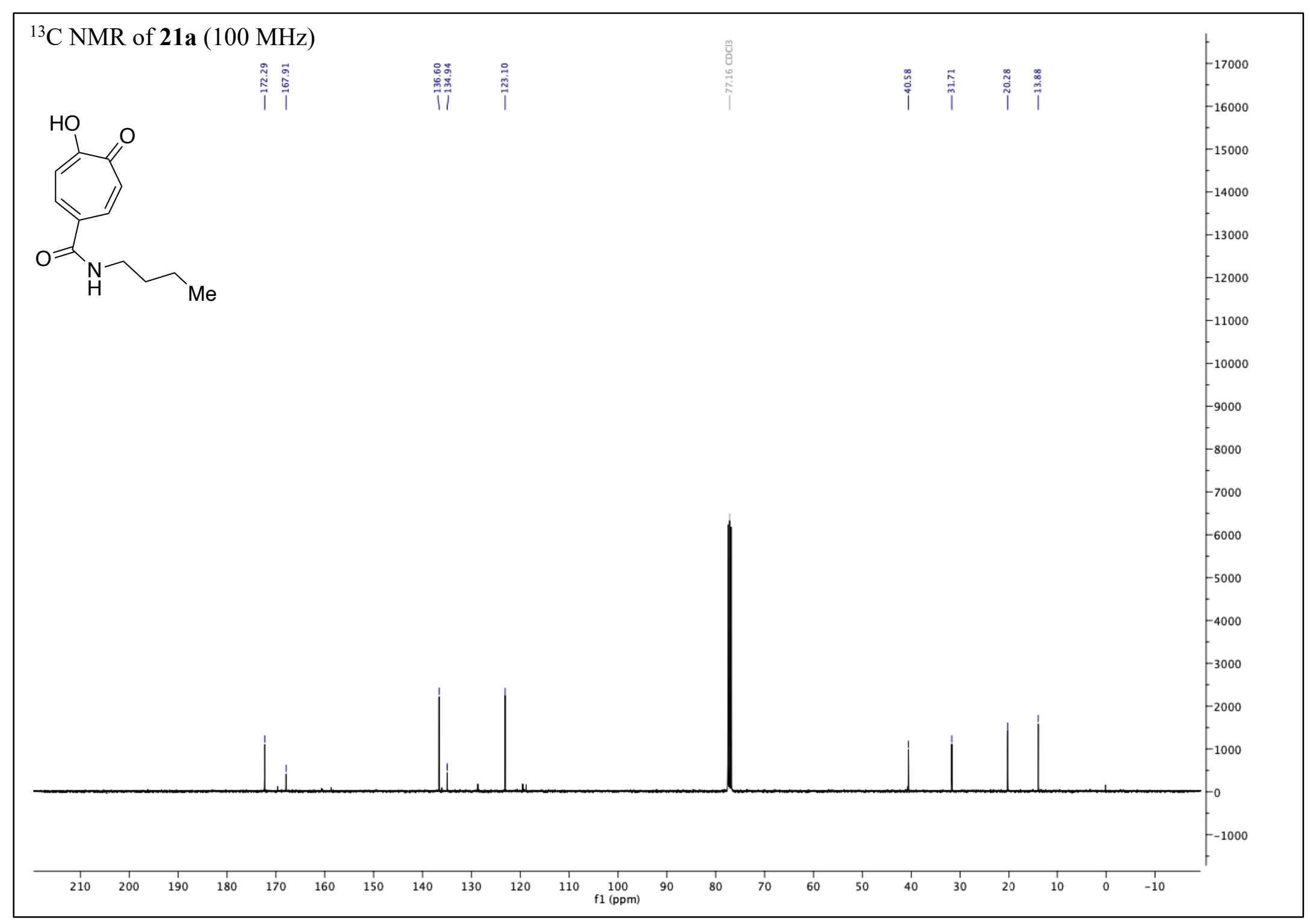


$N$-(2-(3-(but-3-yn-1-yl)-3H-diazirin-3-yl)ethyl)-4-hydroxy-5-oxocyclohepta-1,3,6-triene-1-carboxamide (21b)

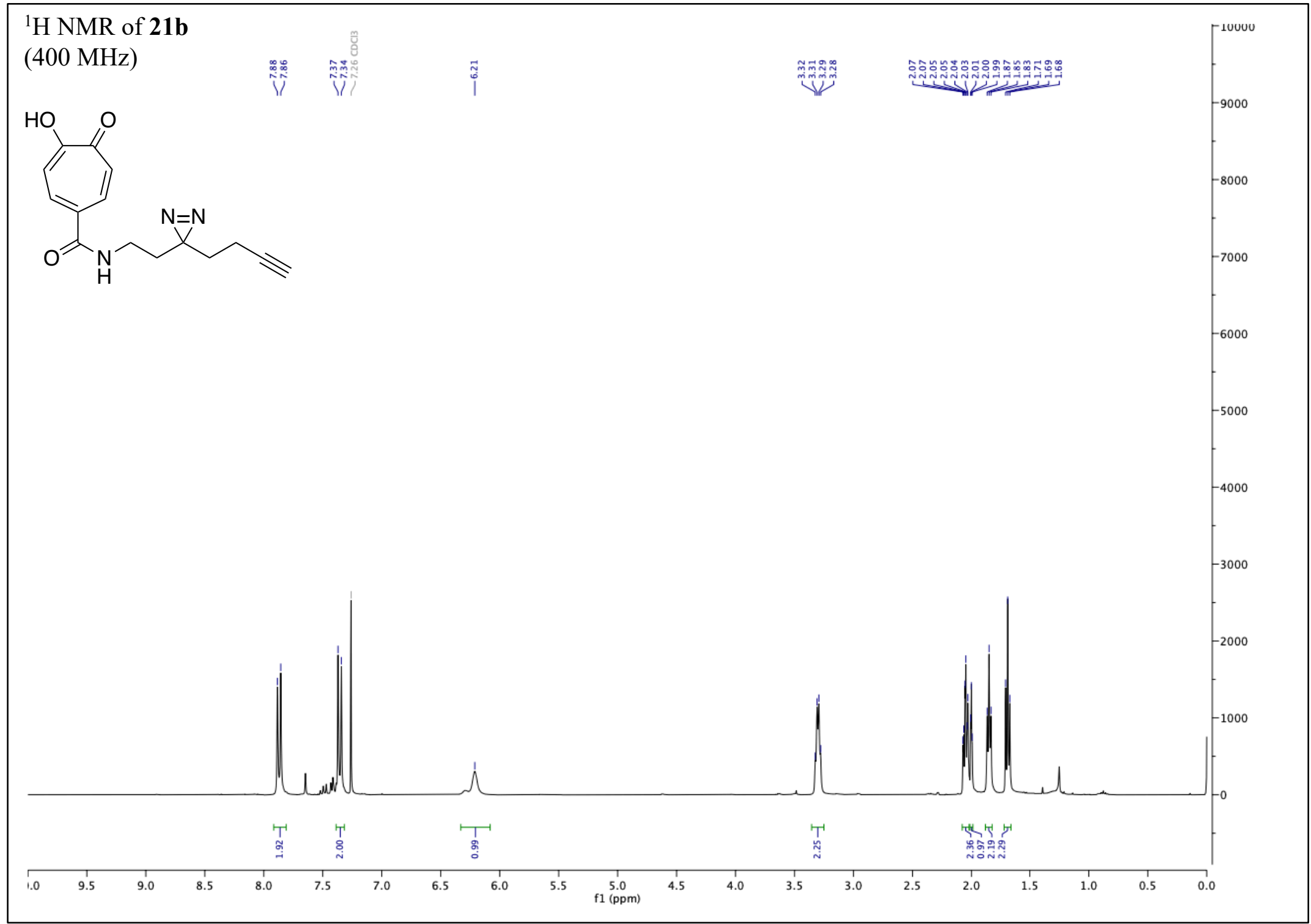




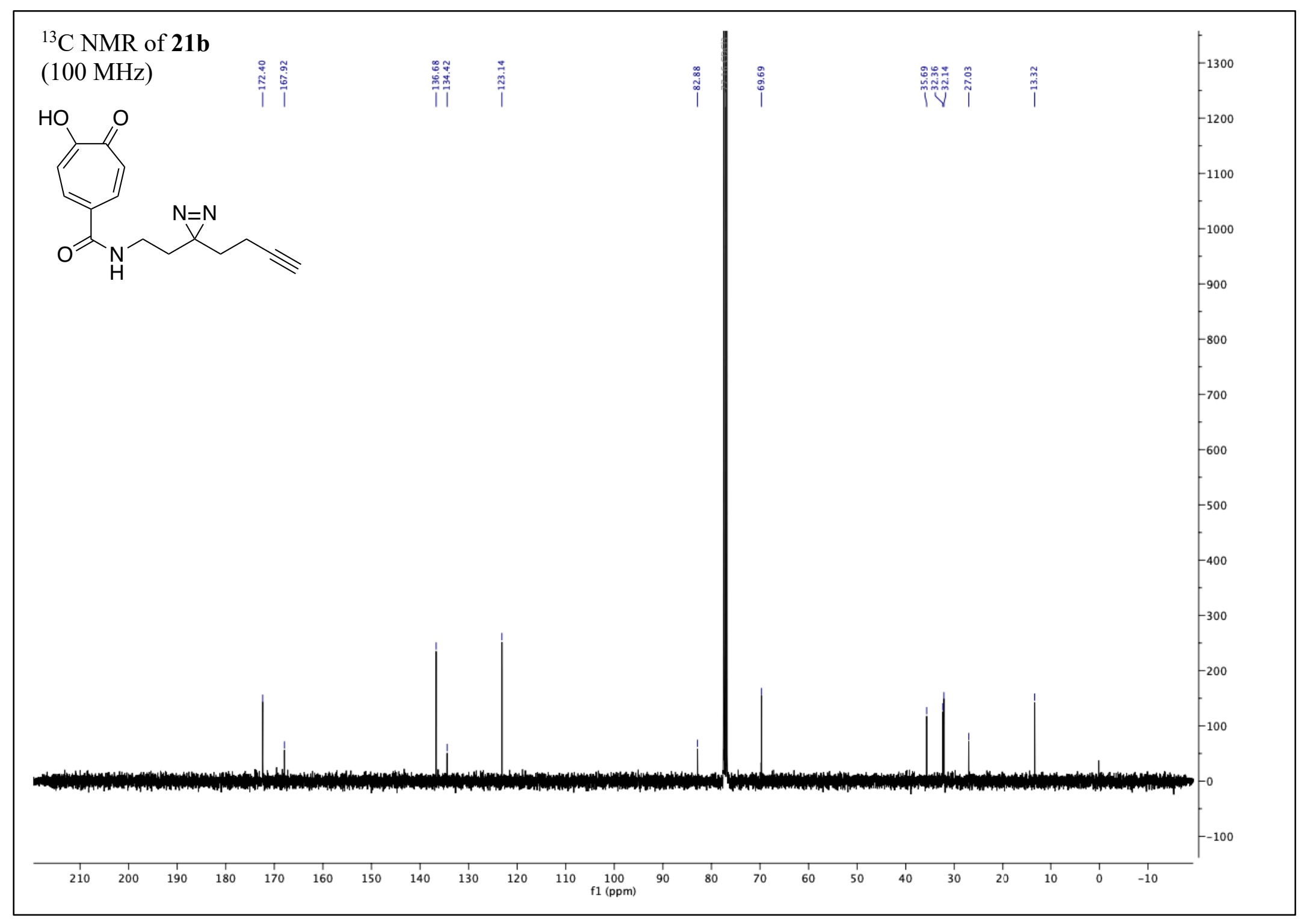


$N$-benzyl-4-hydroxy-5-oxocyclohepta-1,3,6-triene-1-carboxamide (21c)

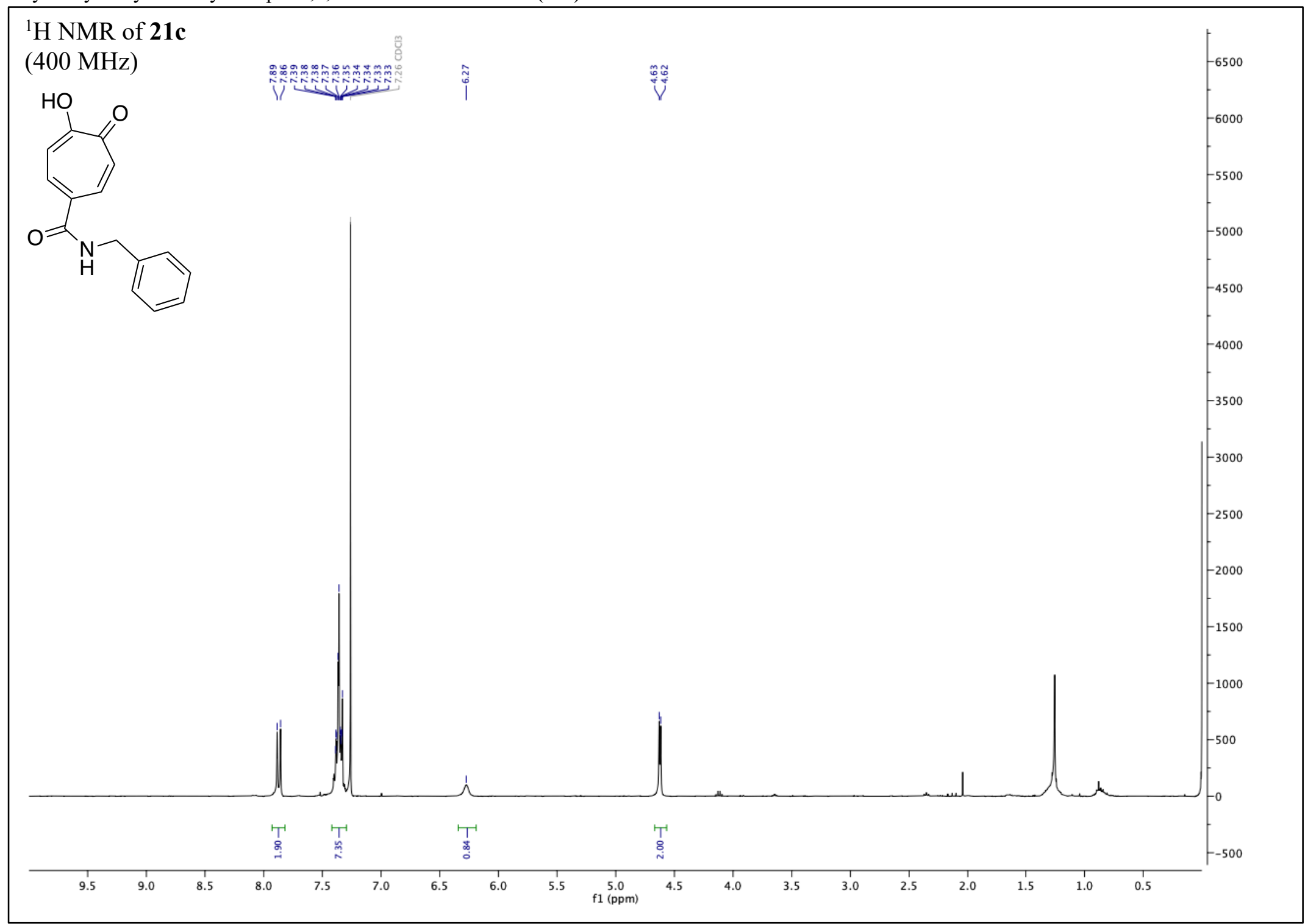




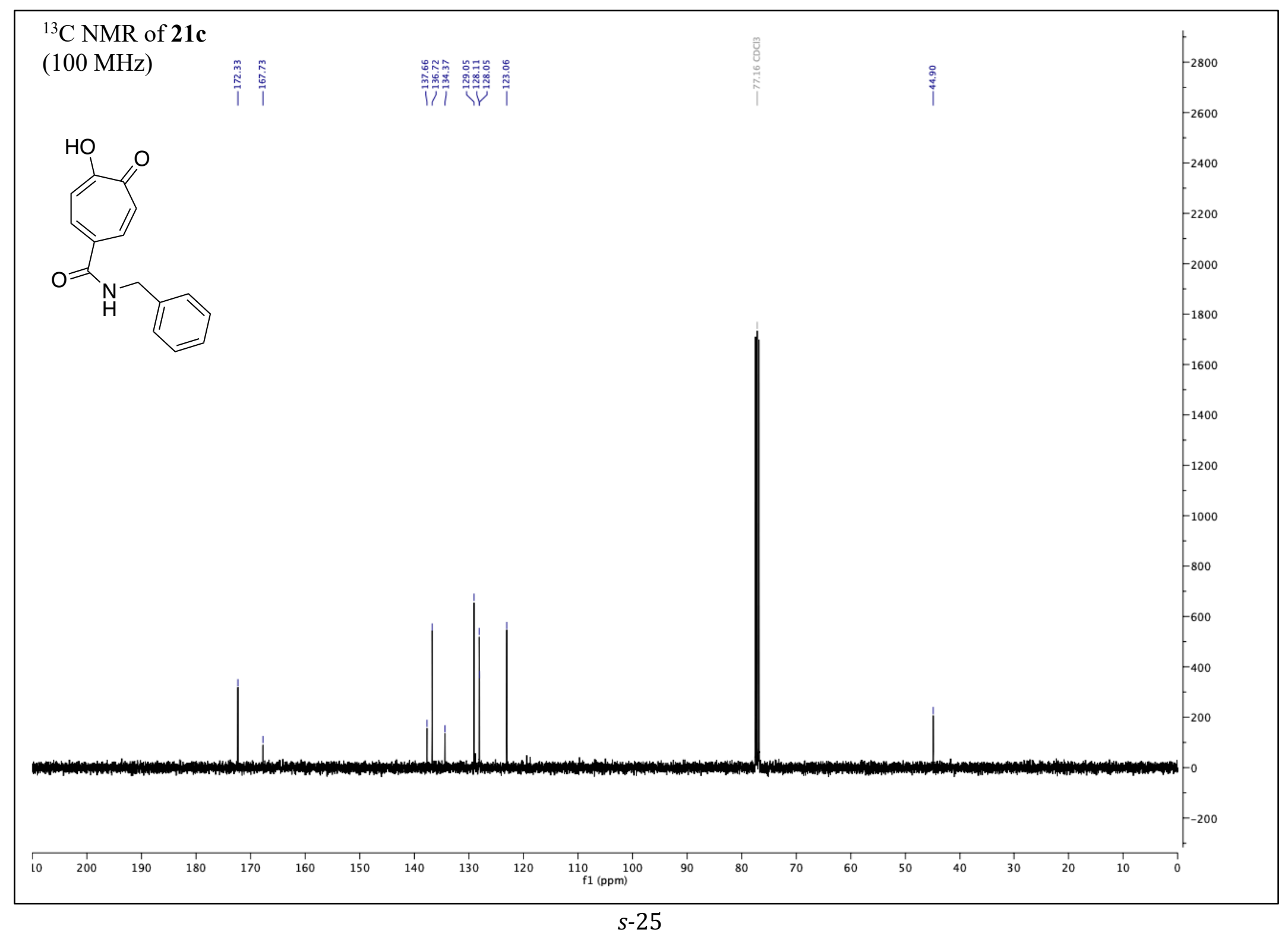


2,4-dihydroxy-5-oxocyclohepta-1,3,6-triene-1-carboxylic acid (26)

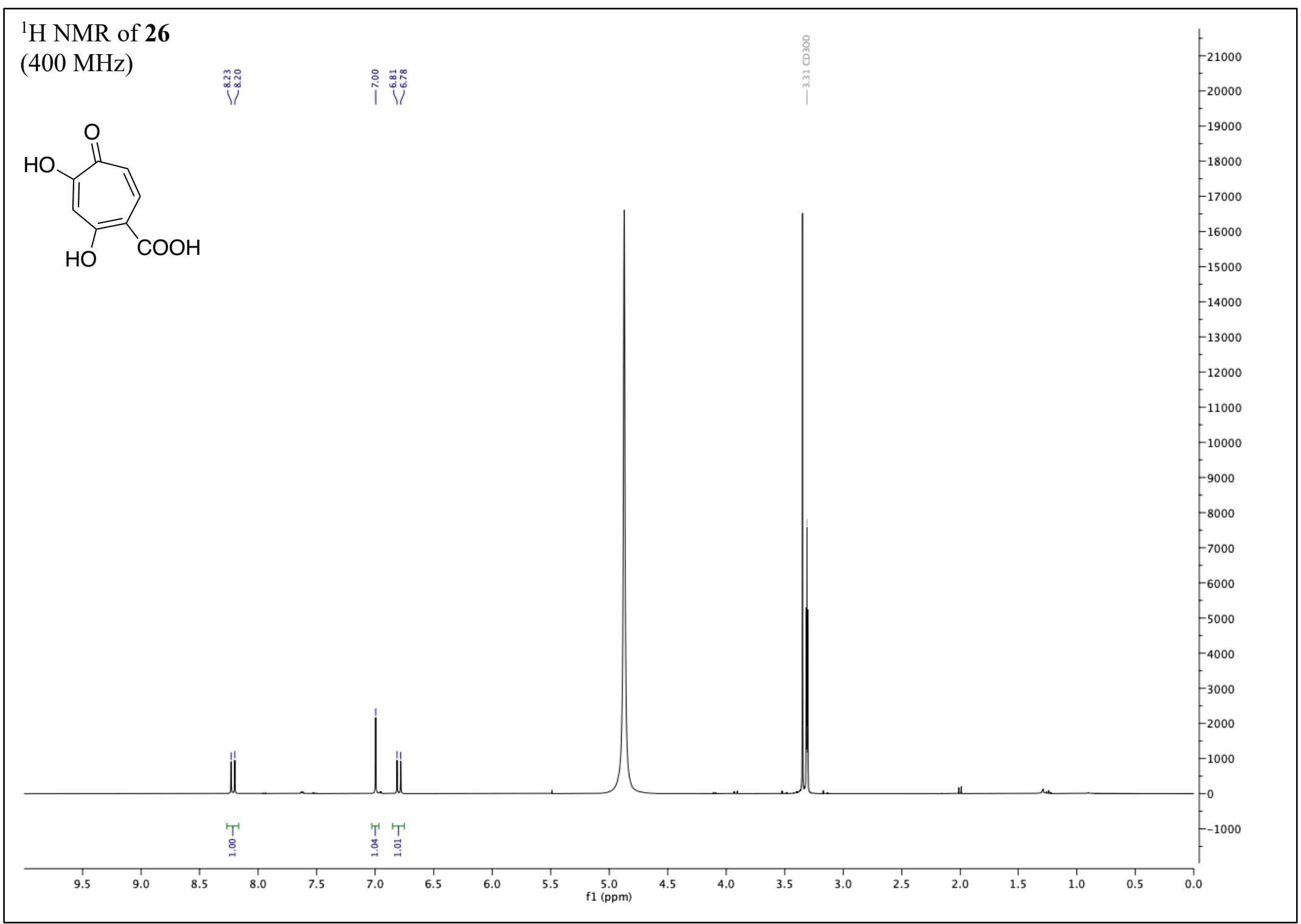




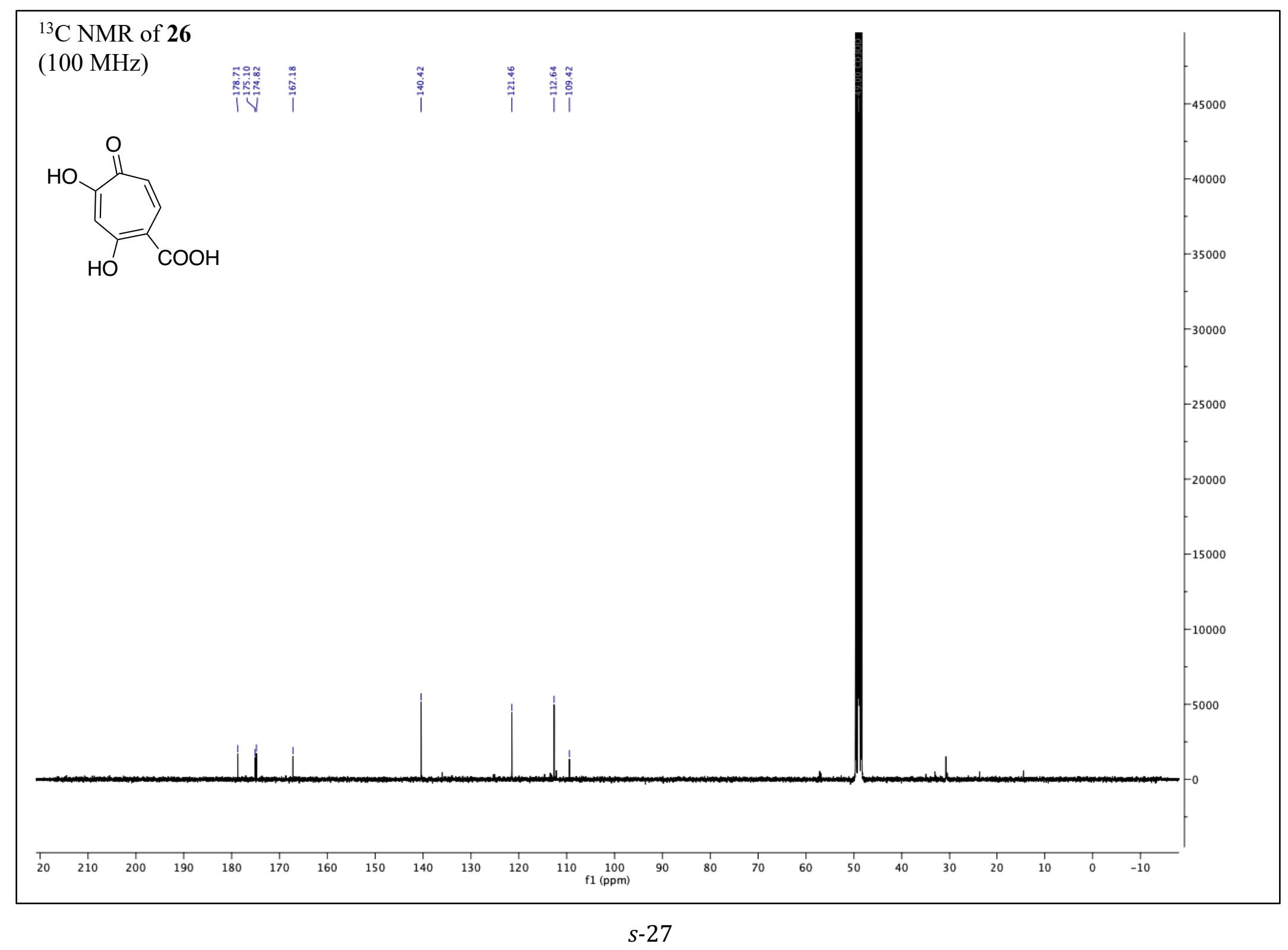


Ethyl 4-hydroxy-3-oxocyclohepta-1,4,6-triene-1-carboxylate (6)

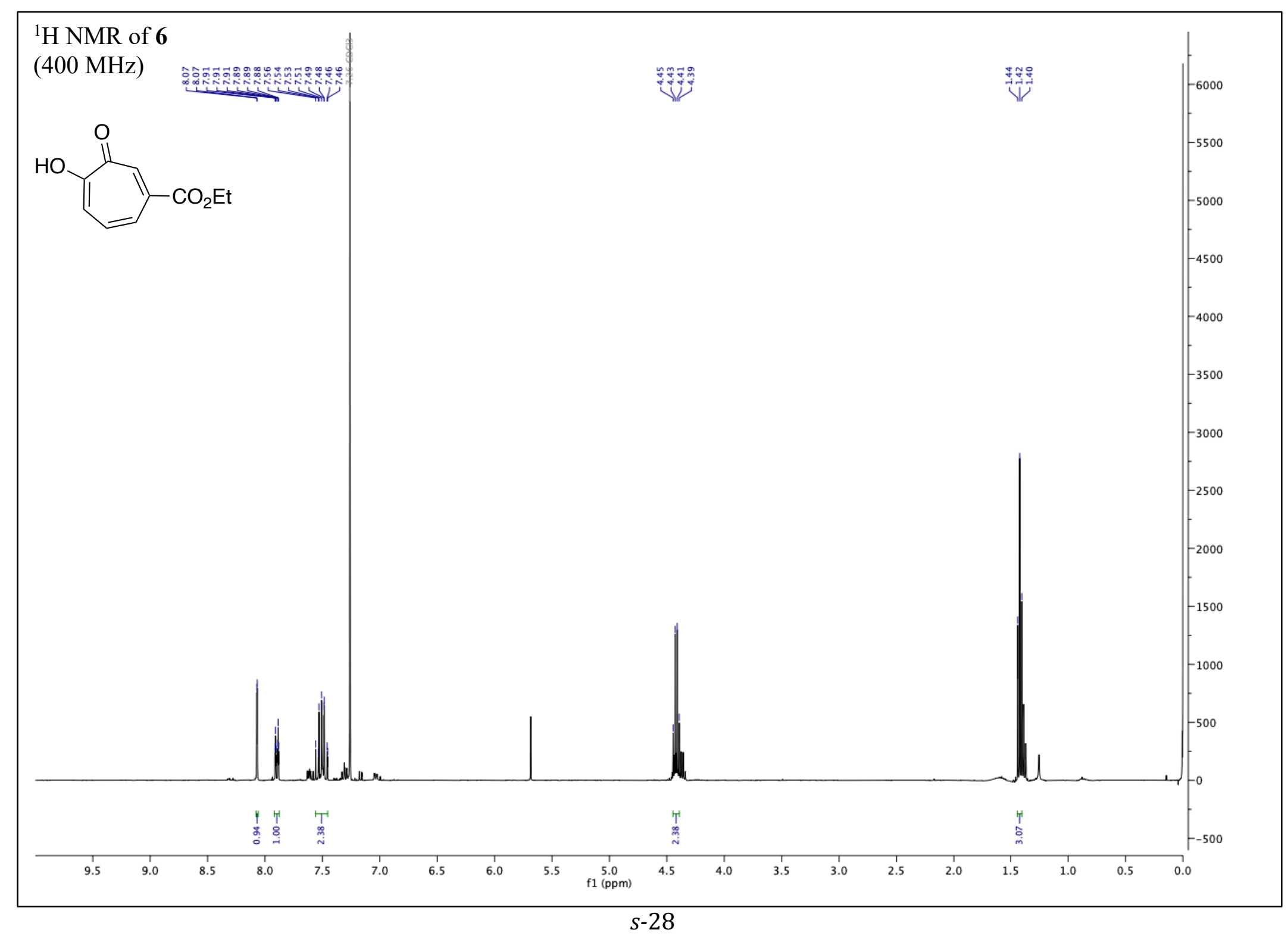




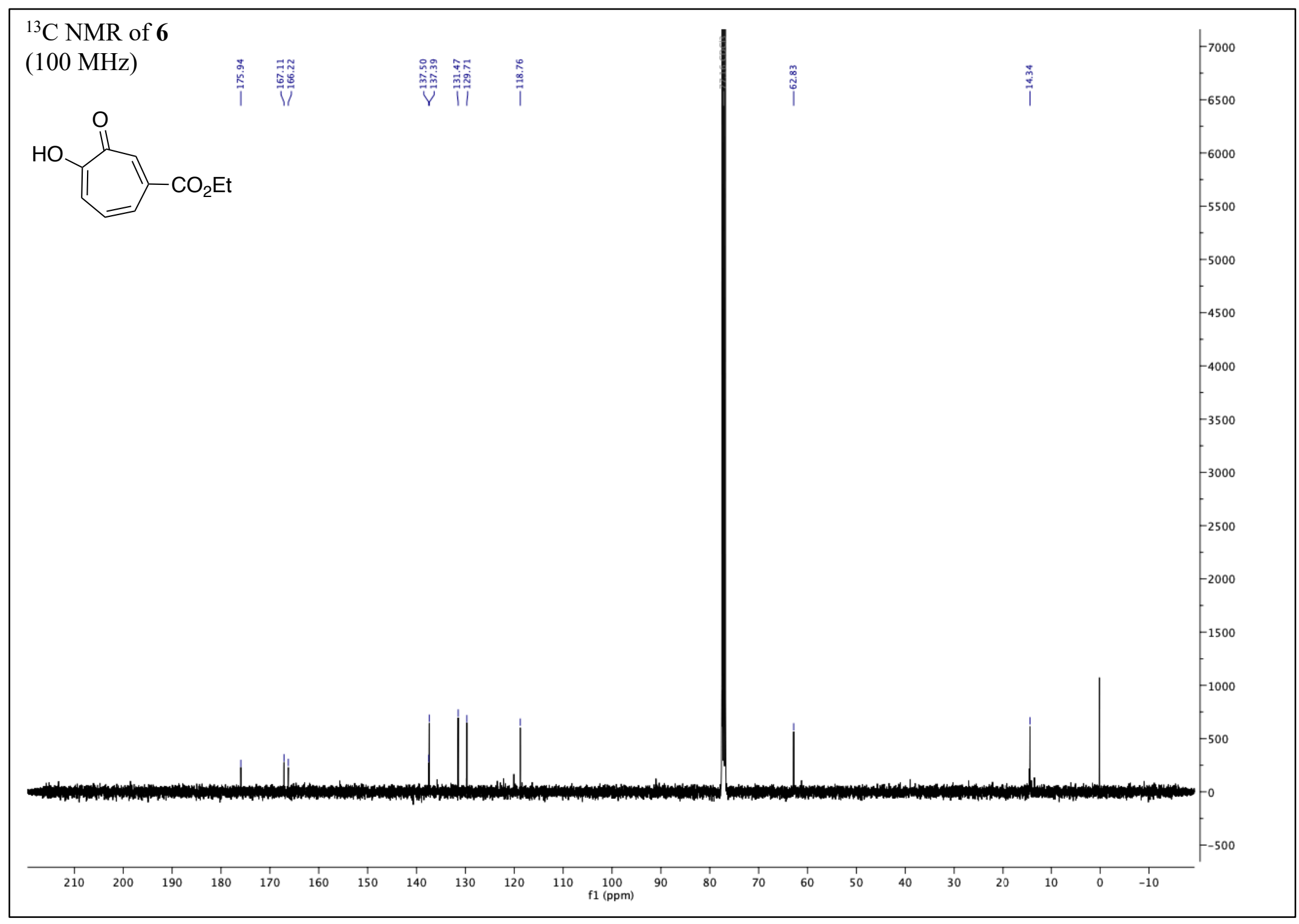

